\title{
A review on the molecular basis underlying the protective effects of Andrographis paniculata and andrographolide against myocardial injury
}

\section{Sok Kuan Wong 1 \\ Kok-Yong Chin \\ Soelaiman Ima-Nirwana}

Department of Pharmacology, Faculty of Medicine, Universiti Kebangsaan Malaysia, Cheras, Kuala Lumpur, 56000, Malaysia
Correspondence: Soelaiman Ima-Nirwana Department of Pharmacology, Faculty of Medicine, Universiti Kebangsaan Malaysia, Jalan Yaacob Latif, Bandar Tun Razak, Cheras, 56000, Kuala Lumpur, Malaysia Tel +62 603-91459579 Fax +62 603-91459545

Email imasoel@gmail.com

\begin{abstract}
Andrographolide is the major compound found in the medicinal plant, Andrographis paniculata (Burm.f.) Nees, which accounts for its medicinal properties. Both the plant extract and compound have been reported to exhibit potential cardiovascular activities. This review summarises related studies describing the biological activities and target mechanisms of $A$. paniculata and andrographolide in vivo and in vitro. The current evidence unambiguously indicated the protective effects provided by A. paniculata and andrographolide administration against myocardial injury. The intervention ameliorates the symptoms of myocardial injury by interfering with the inductive phase of a) inflammatory response mediated by nuclear factor-kappa B (NF- $\kappa \mathrm{B})$, phosphatidylinositol 3-kinase (PI3K)/protein kinase B (Akt), mitogen-activated protein kinase (MAPK) and signal transducer and activator of transcription 3 (STAT3) signalling molecules; b) oxidative stress via activation of nuclear factor erythroid 2-related factor (Nrf-2) and reduction of enzymes responsible for generating reactive oxygen and nitrogen species; c) intrinsic and extrinsic mechanisms in apoptosis regulated by upstream insulinlike growth factor-1 receptor (IGF-1R) and peroxisome proliferator-activated receptoralpha (PPAR- $\alpha$ ); d) profibrotic growth factors thus reducing cardiac fibrosis, improving endothelial function and fibrinolytic function. In conclusion, A. paniculata and andrographolide possess therapeutic potential in the management of myocardial injury, which requires further validation in human clinical trials.
\end{abstract}

Keywords: Andrographis, myocardial infarction, inflammation, oxidative stress, apoptosis, fibrosis

\section{Introduction}

Myocardial injury is the damage to myocardium affecting the heart function. It can be detected by the elevation of cardiac troponin concentration exceeding the 99th percentile of the reference population or the upper reference limit. ${ }^{1}$ It can be classified into acute and chronic conditions. Acute myocardial injury refers to dynamic increase and/or decrease of troponin concentration, whereas chronic myocardial injury is characterised by persistent elevated troponin concentration. Both conditions can result from cardiovascular or non-cardiovascular factors in clinical practice. ${ }^{1}$ Treatment strategies for type I myocardial infarction are well established, but there is no clinical guidance or recommended therapy for patients with other myocardial injury categories. ${ }^{2,3}$ These shortcomings prompt the search for alternative agents to manage myocardial injury. 
Andrographis paniculata (Burm.f.) Nees is a herbaceous plant traditionally used to treat rheumatoid arthritis, diarrhoea, upper respiratory tract infection, laryngitis, fever, and cancer in China, India, and Southeast Asia. ${ }^{4}$ It is also generally known as "the king of bitters" due to its extremely bitter taste. The local name in Malaysia is "hempedu bumi", loosely translated as "earthly bile", reflecting its bitter taste. Andrographolide (Figure 1) is a natural diterpenoid lactone extracted from A. paniculata (Burm.f.) Nees. Scientific research revealed that andrographolide is the phytoconstituent accounts for the medicinal properties of $A$. paniculata (Burm.f.) Nees. Both plant extract and its bioactive compound are proven through pharmacological research to possess extensive biological effects, such as anti-neoplasm, ${ }^{5-7}$ anti-bacteria, ${ }^{8,9}$ anti-malaria, ${ }^{10,11}$ hepatoprotection, ${ }^{12,13}$ antiinflammation, ${ }^{10,13-15}$ anti-oxidant, ${ }^{15}$ anti-apoptosis, ${ }^{16,17}$ anti-fibrosis, ${ }^{14,18}$ and cardioprotection. ${ }^{19}$ Among these, the latter three bioactivities are closely associated with the management of myocardial injury.

Increasing evidence demonstrated the potential beneficial use of $A$. paniculata (Burm.f.) Nees in humans against respiratory tract infections, ${ }^{20}$ diabetes, ${ }^{21}$ inflammatory bowel diseases, ${ }^{22}$ fatigue reduction in multiple sclerosis, ${ }^{23}$ osteoarthritis, ${ }^{24}$ common cold, ${ }^{25}$ and hypertriglyceridaemia. ${ }^{26}$

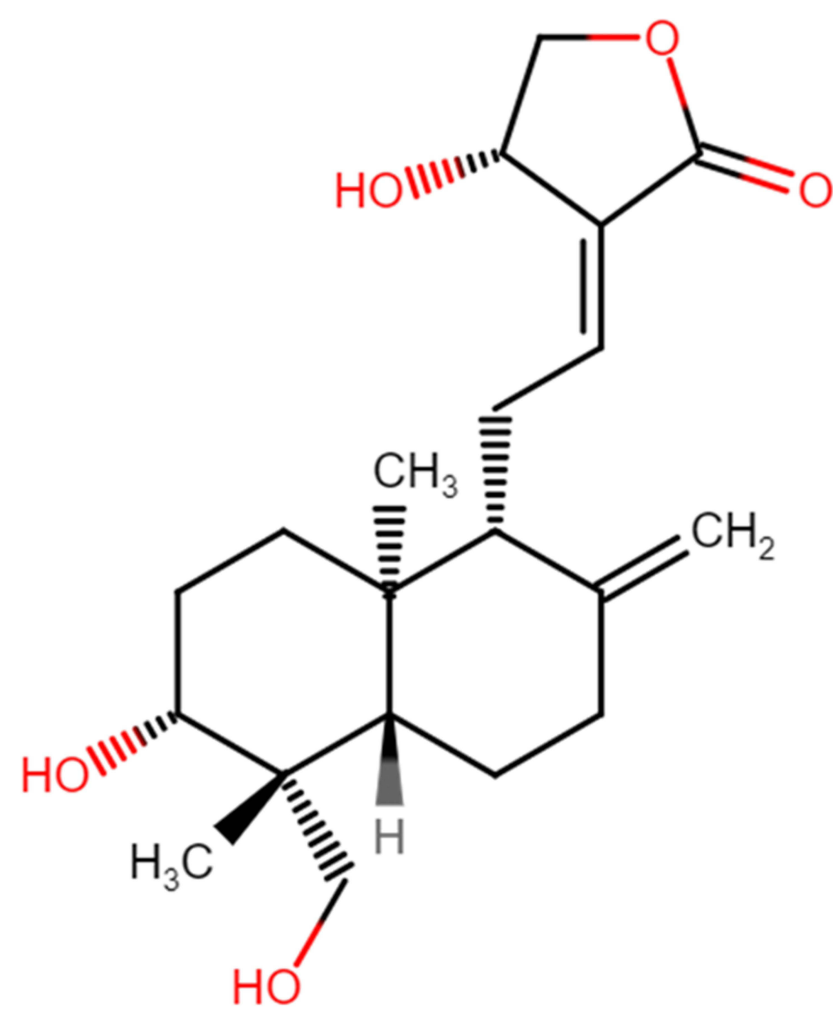

Figure I The chemical structure of andrographolide.
The doses of $A$. paniculata (Burm.f.) Nees used in these human trials range from 300 to $1800 \mathrm{mg} /$ day. Clinical trials indicated that andrographolide is a promising therapy for multiple sclerosis, ${ }^{27}$ rheumatoid arthritis, ${ }^{28}$ as well as hand, foot, and mouth diseases. ${ }^{29}$ Much lower doses of andrographolide than A. paniculata (Burm.f.) Nees have been tested, varying from 5 to $140 \mathrm{mg} /$ day. In addition, an array of studies reported that andrographolide and its original plant source, A. paniculata (Burm.f.) Nees are generally non-toxic when taken orally. ${ }^{30,31}$ For these reasons, studies on the pharmacological functions of andrographolide are still in progress today.

With the presence of diverse pharmacological effects in andrographolide and A. paniculata (Burm.f.) Nees, particularly in reducing cardiac fibrosis and improving cardiac function, it is imperative to review and consolidate current evidence on their beneficial effects against myocardial injury. The molecular mechanism underlying their protective effects are also scrutinised. To date, the investigation on the effects of A. paniculata (Burm.f.) Nees and andrographolide in patients with myocardial injury is limited. This review provides an overview of the pharmacological properties that could promote the development and application of andrographolide and A. paniculata (Burm.f.) Nees as prophylactic or therapeutic agents for myocardial injury.

\section{General Isolation of Andrographolide from A. paniculata (Burm.f.) Nees}

Andrographolide has been extracted from A. paniculata (Burm.f.) Nees by researchers using different types of solvents, including dichloromethane, methanol, ethanol, and toluene. A rapid isolation method was developed two decades ago using a mixture of dichloromethane and methanol at 1:1 ratio, methanol, or $95 \%$ alcohol as solvents. The extracts were filtered and solvents were removed under vacuum. ${ }^{32}$ Pundarikakshudu et al employed five simple methods for A. paniculata (Burm.f.) Nees isolation. The three isolation methods used toluene as solvent for cold maceration, followed by refluxing in a mixture of dichloromethane and methanol (1:1), methanol, or ethanol. Another two isolation methods were conducted with maceration in methanol or mixture of dichloromethane and methanol (1:1). The extracts obtained from these approaches were filtered and evaporated to form solid crystalline mass. ${ }^{33}$ Recently, commercial Andrographis products were used to obtain their methanolic extracts. The product was soaked in methanol, sonicated at room temperature for 60 minutes, filtered through filter 
paper, and evaporated under vacuum. ${ }^{34}$ Analysis of thinlayer or high-performance liquid chromatography detected the presence of andrographolide in all these extracts. ${ }^{32-34}$ In general, the extraction protocol was initiated with maceration of leaf powder in solvent, followed by filtration through filter paper. Next, the solution was evaporated under reduced pressure to yield the green-colour powdered crude extract containing andrographolide.

\section{Literature Search}

Literature search was performed using PubMed and Scopus search engines. The keywords used are "(Andrographis paniculata OR andrographolide) AND (myocardial injury OR myocardial infarction OR ischaemia OR endothelial dysfunction OR cardiomyocyte)". The search identified 17 articles in PubMed and 69 articles in Scopus databases from inception until February 15, 2021. All articles were written in English. We excluded duplicate articles $(n=13)$ retrieved from two search engines. The remaining search results were screened by referring to the title, abstract, and full-text. We included in vitro and in vivo studies demonstrating the effects and mechanism of action of $A$. paniculata (Burm.f.) Nees and andrographolide on myocardial injuries. The screening of the search results excluded 26 review articles and 32 irrelevant articles not fulfilling the inclusion criteria. A total of 15 original research articles were included in this review (Figure 2).

\section{Nomenclature of the Plant Extract}

The accurate nomenclature of plants is crucial in all scientific studies reporting the pharmacological effects of plants to avoid ambiguities and error. ${ }^{35}$ A total of seven studies in this review examined the effects of $A$. paniculata (Burm.f.) Nees against myocardial injuries in animals. ${ }^{17,36-41}$ However, there was only one study performed the

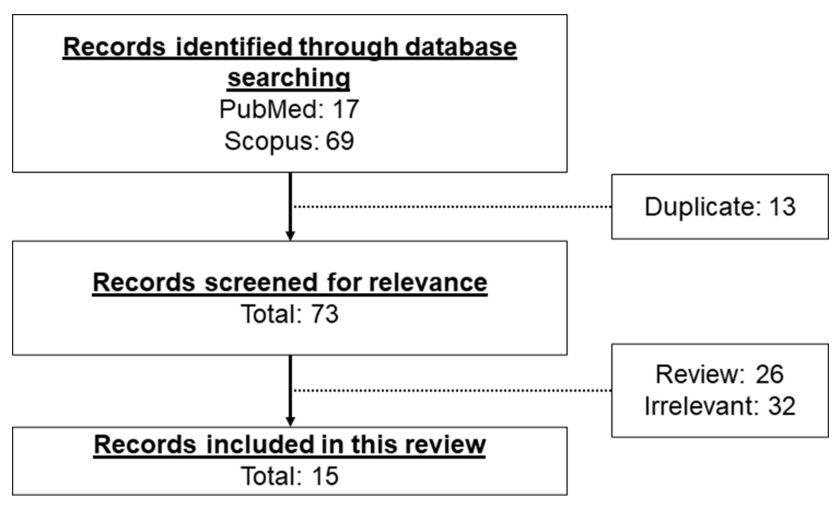

Figure $\mathbf{2}$ The framework for the selection of relevant studies. verification of plant extract by one of the researchers in their group and the specimen has been deposited in herbarium with a voucher number. ${ }^{17}$ The correct nomenclature of the plant taxonomically is "A paniculata (Burm.f.) Nees". This plant is categorised under the family Acanthaceae Juss. and genus Andrographis Wall. ex Nees. Hence, the name of the plant has been written correctly in this review.

\section{Effects of Andrographolide on Myocardial Injury in Animals}

A. paniculata (Burm.f.) Nees plant extract or andrographolide increased mean arterial pressure (MAP), heart rate, and improved myocardial function in animals. Left ventricular ejection fraction (LVEF), fractional shortening (LVFS), endsystolic pressure (LVESP), early to late mitral flow (E/A ratio), rate of intra-ventricular pressure rise and decline $( \pm \mathrm{dP} / \mathrm{dt})$, and cardiac output were increased, whereas left ventricular enddiastolic pressure (LVEDP), internal dimension in systole (LVDS), end systolic diameter (LVESd), end diastolic diameter (LVEDd), posterior wall dimension (LVPWd), and infarction size were decreased ${ }^{18,36,40-44}$ after administration of A. paniculata (Burm.f.) Nees extract or andrographolide. Histological assessment revealed that the pathological changes of heart tissue were repaired after treatment of $A$. paniculata (Burm.f.) Nees or andrographolide (as seen by reduced interstitial spaces, fibrosis, myocardial degeneration, distortion in the cardiomyocyte arrangement, cell oedema, degree of necrosis, and neutrophil infiltration). ${ }^{17,39-41,45,46}$ Administration of A. paniculata (Burm.f.) Nees or andrographolide also reversed cardiac fibrosis [indicated by lowered fibrosis marker such as alpha-smooth muscle actin ( $\alpha$-SMA)] and cardiac hypertrophy [cross-sectional area of cardiomyocytes, heart weight normalised with tibia length or body weight, atrial natriuretic peptide (ANP), brain natriuretic peptide (BNP), and beta-myosin heavy chain were reduced]. ${ }^{17,18,43-45}$ Malignant arrhythmia was absent, ischaemic electrocardiogram and damage to myocardial ultrastructure were milder in mongrel dogs with myocardial ischemia and reperfusion injury receiving $A$. paniculata (Burm.f.) Nees extract. ${ }^{36,37}$ The changes in myocyte-specific enzymes, such as creatine phosphokinase-MB (CK-MB) and lactate dehydrogenase, were also noted with $A$. paniculata (Burm.f.) Nees treatment. ${ }^{40,41}$ Survival rate was improved in a rat model of autoimmune myocarditis induced by Mycobacterium tuberculosis-supplemented porcine cardiac myosin ${ }^{43}$ and myocardial infarction induced by left coronary artery ligation $^{18}$ upon administration of andrographolide. 
In summary, A. paniculata (Burm.f.) Nees extract or andrographolide has been consistently reported for its protection against myocardial injury using various types of animal models (Table 1). Based on the accumulated studies, the reported effective doses for A. paniculata (Burm. f.) Nees plant extract against myocardial injury ranged from 100 to $2000 \mathrm{mg} / \mathrm{kg}$ with a treatment duration of $7-$ 31 days. For andrographolide, the effective dose ranged from 10 to $100 \mathrm{mg} / \mathrm{kg}$ with a treatment duration of $1-12$ weeks in animal models. A shorter duration may be sufficient if a higher dose is administered, while a longer treatment duration may be required if lower dose is supplemented. However, their effects in reversing myocardial injury in humans are yet to be investigated. The efficacy of A. paniculata (Burm.f.) Nees or andrographolide need to be further assessed in clinical trial before any conclusion can be drawn.

\section{Mechanism of Action Underlying the Protective Effects of A. paniculata (Burm.f.) Nees and Andrographolide Against Myocardial Injury}

Researchers have uncovered the mechanism of action for A. paniculata (Burm.f.) Nees and andrographolide as potential cardioprotective agents through preclinical studies (Tables 1 and 2). The bioactivities of $A$. paniculata (Burm.f.) Nees and andrographolide include the suppression of inflammatory response, oxidative stress, apoptosis, cardiac fibrosis, and endothelial dysfunction.

\section{Regulation of Inflammatory Response}

Inflammation is the hallmarks of myocardial injury. Macrophages play an important role in inflammation. They are involved in antigen presentation, phagocytosis, and immunomodulation via the release of cytokines and chemokines. ${ }^{47}$ Various clusters of differentiation are expressed on the surface of macrophages. Cluster of differentiation 3 (CD3) is a T-cell co-receptor that activates both cytotoxic and helper T cells. ${ }^{48}$ Cluster of differentiation 14 (CD14) forms a multi-receptor complex with Tolllike receptor for the recognition of lipopolysaccharides (LPS) ${ }^{49,50}$ Cluster of differentiation 45 (CD45) is a hematopoietic cell marker that indicates the activation of one or more inflammatory cell types. ${ }^{51}$ Cluster of differentiation 68 (CD68) is a common histochemical/cytochemical marker of inflammation whereby the upregulation is a response of exposure to inflammatory stimuli, such as oxidised low-density lipoprotein (oxLDL). ${ }^{52}$ Cluster of differentiation 86 (CD86) provides initial co-stimulatory signals for T-cell activation. ${ }^{53,54}$ The increased expression of these cluster of differentiation activates pro-inflammatory signalling pathways, thereby inducing the secretion of inflammatory cytokines. On the other hand, cluster of differentiation 206 (CD206) involves in the resolution of inflammation. The ligation of CD206 produces anti-inflammatory response by inhibiting proinflammatory agents and reactive oxygen species (ROS). ${ }^{55}$

The initiation and progression of inflammatory response are tightly regulated by a series of signalling molecules, including nuclear factor-kappa B (NF-кB), phosphatidylinositol 3-kinase (PI3K)/protein kinase $\mathrm{B}$ (Akt), and mitogen-activated protein kinase (MAPK). The link between diverse external cell stressors and expression of inflammatory cytokines is mediated through phosphorylation of inhibitor of NF- $\mathrm{KB}(\mathrm{I} \kappa \mathrm{B} \alpha)$ resulting in its ubiquitination and dissociation from NF- $\mathrm{BB}$. The nuclear translocation of activated NF- $\mathrm{kB}$ initiates the expression of inflammatory mediators. ${ }^{56}$ The PI $3 \mathrm{~K} / \mathrm{Akt}$ pathway is triggered by the engagement of receptor tyrosine kinase or Toll-like receptor with their respective ligands. PI $3 \mathrm{~K}$ is recruited and activated, facilitating the conversion of phosphatidylinositol (3,4)-bisphosphate $\left(\mathrm{PIP}_{2}\right)$ to phosphatidylinositol $(3,4,5)$-trisphosphate $\left(\mathrm{PIP}_{3}\right)$ and subsequent phosphorylation of Akt. Eventually, the activated Akt promotes inflammatory response. ${ }^{57}$ Similarly, increased MAPK activity and the crosstalk between MAPK and NF- $\kappa B$ further enhances the transcription and translation of inflammatory mediators. ${ }^{58}$ Since the activation of NF- $\mathrm{KB}, \mathrm{PI} 3 \mathrm{~K} / \mathrm{Akt}$, and MAPK is involved in modulating an inflammatory response, the targeted therapeutic strategies aiming at inhibition of these signalling molecules could prevent excessive inflammation.

The anti-inflammatory response has been reported in A. paniculata (Burm.f.) Nees extract, conferring protection against pathological cardiac hypertrophy. Apart from causing NF-kB inhibition, oral administration of $A$. paniculata (Burm.f.) Nees extract suppressed MAPK signalling pathway to mitigate inflammation. The level of IL-6 was decreased and its associated downstream signalling molecules [signal transducer and activator of transcription 3 (STAT3), mitogen/extracellular signal-regulated kinase kinase-5 (MEK5), dual-specificity mitogen/extracellular signal-regulated kinase kinase (MEK1/2) and c-Jun N-terminal kinase (JNK)] were inhibited. ${ }^{17}$ 


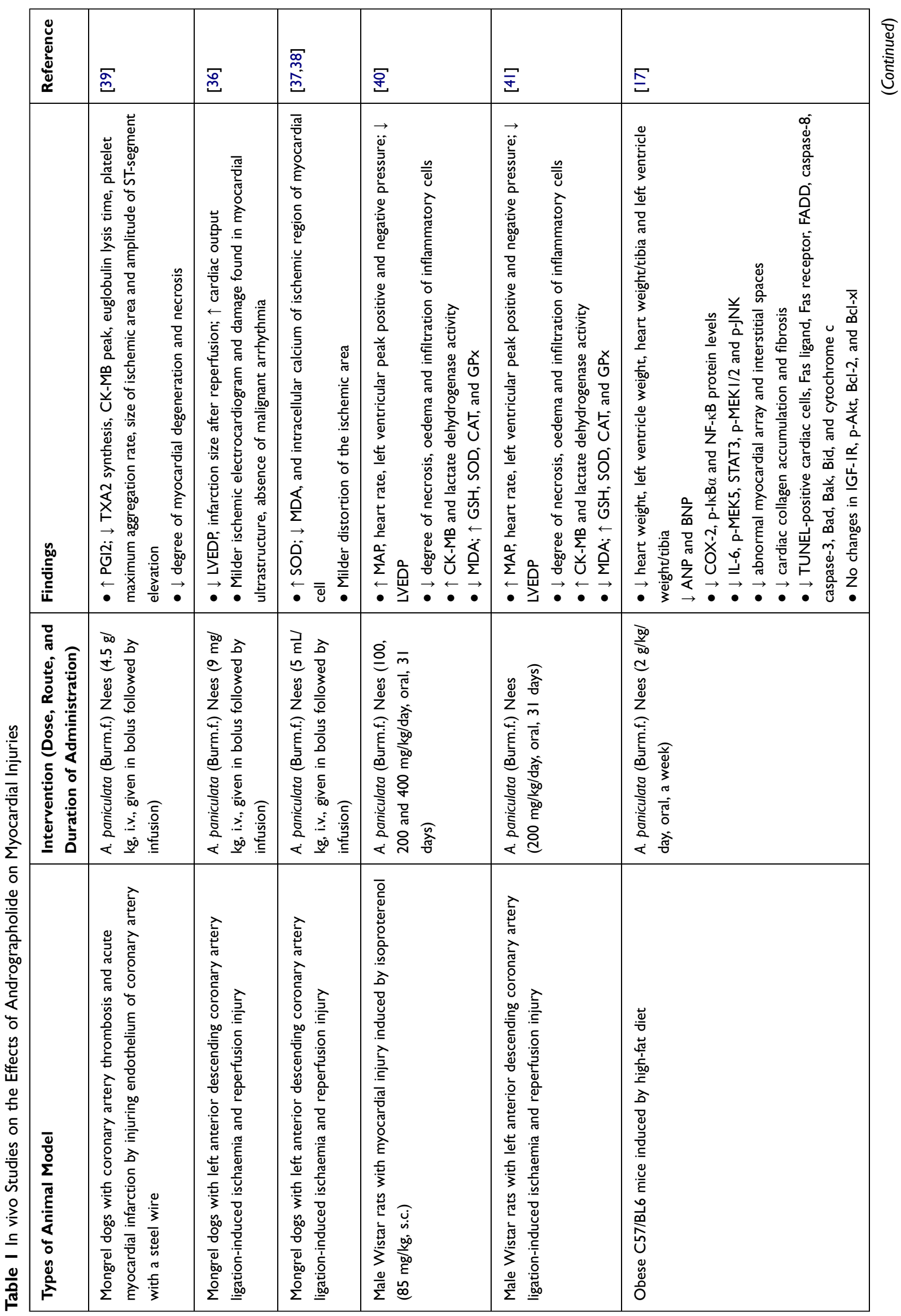




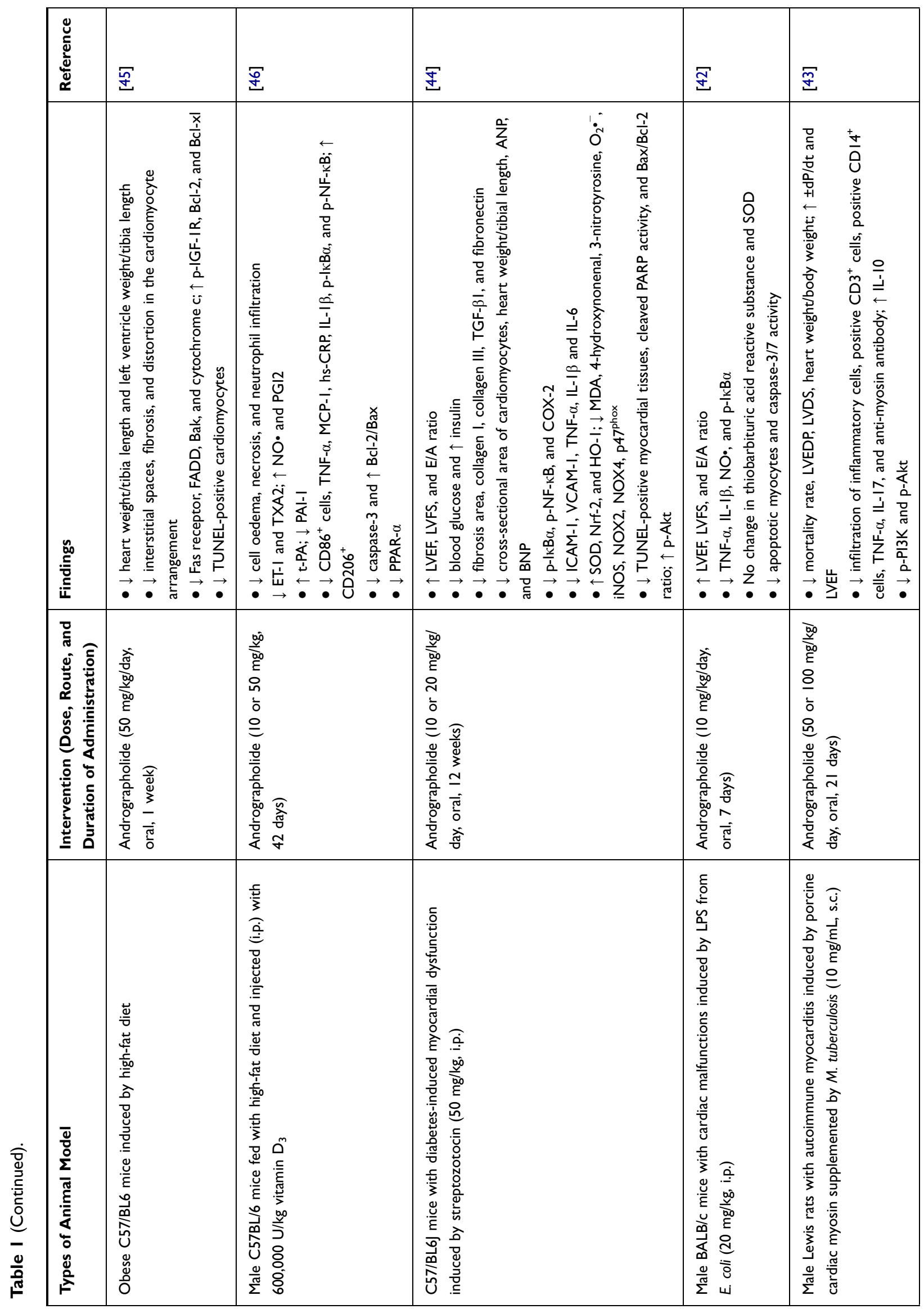




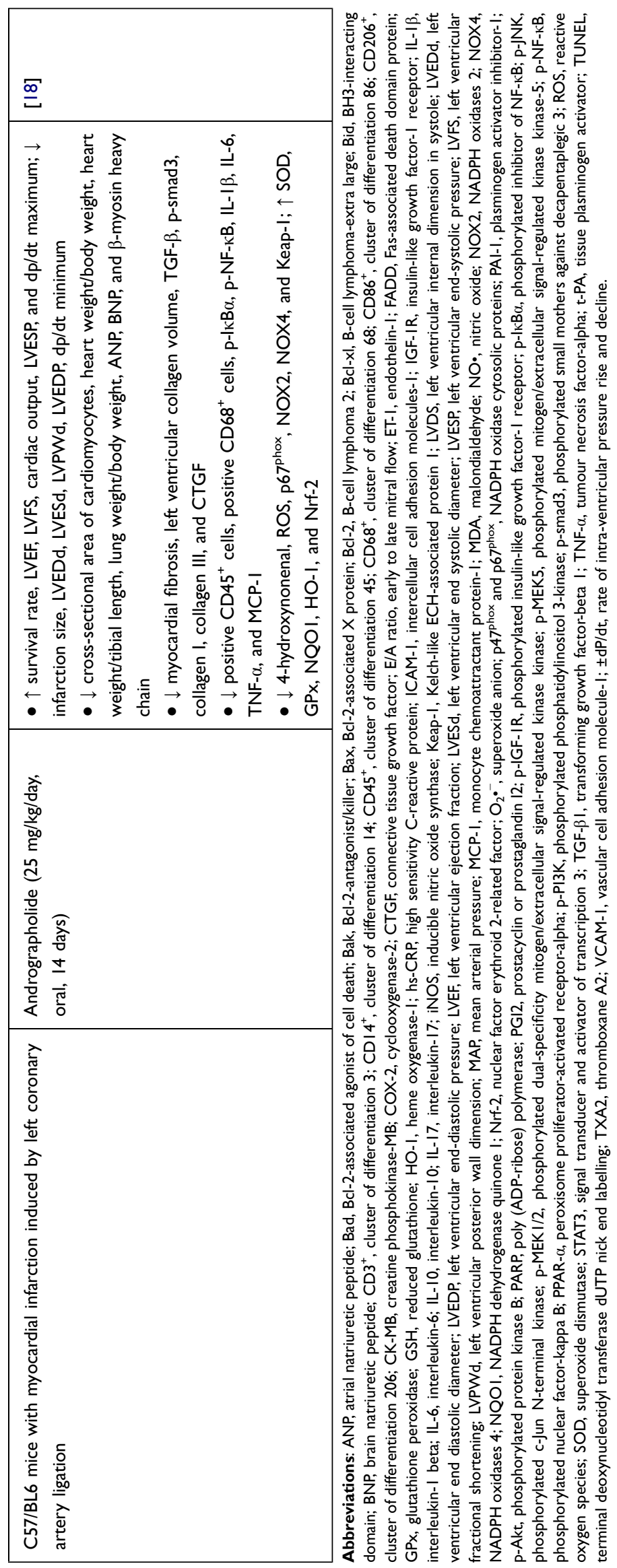


Table 2 In vitro Studies on the Effects of Andrographolide on Cardiomyocytes

\begin{tabular}{|c|c|c|c|}
\hline Types of Cell & Intervention & Findings & Reference \\
\hline $\begin{array}{l}\text { Primary rat myocardial microvascular } \\
\text { endothelial cells stimulated by LPS }\end{array}$ & Andrographolide $(10 \mu \mathrm{g} / \mathrm{mL})$ & $\begin{array}{l}\text { - No cytotoxicity } \\
\text { - } \downarrow \text { CAVI, IL-6 and TNF- } \alpha\end{array}$ & [59] \\
\hline $\begin{array}{l}\mathrm{H} 9 \mathrm{C} 2 \text { cardiomyocytes stimulated by high } \\
\text { concentration of glucose }\end{array}$ & Andrographolide $(\mathrm{I}-10 \mu \mathrm{M})$ & $\begin{array}{l}\text { - } \downarrow \text { ANP and BNP } \\
\text { - } \downarrow \mathrm{p}-\mathrm{I} \mathrm{B} \alpha \text { and } \mathrm{p}-\mathrm{NF}-\kappa \mathrm{B} \\
\text { - } \downarrow \text { ROS, NOX2, NOX4, p47 } \\
2 \text { nuclear translocation } \\
\text { - } \downarrow \text { Bax/Bcl-2 ratio and caspase-3 activity }\end{array}$ & [44] \\
\hline $\mathrm{H} 9 \mathrm{C} 2$ cardiomyocytes subjected to hypoxia & Andrographolide $(12.5-100 \mu \mathrm{M})$ & $\begin{array}{l}\bullet \downarrow \alpha \text {-SMA, TGF- } \beta, \text { p-smad3, collagen I, } \\
\text { fibronectin, and CTGF } \\
\bullet \downarrow \text { ROS, Keap-I, p67 phox, and NOX2; } \uparrow \\
\text { SOD, NQOI, Nrf-2, and HO-I }\end{array}$ & {$[18]$} \\
\hline $\begin{array}{l}\text { Primary neonatal rat cardiomyocytes subjected } \\
\text { to hypoxia and reoxygenation injury }\end{array}$ & Andrographolide $(\mathrm{I}-10 \mu \mathrm{M})$ & $\begin{array}{l}\text { - } \downarrow \text { lactate dehydrogenase } \\
\text { - } \uparrow \text { SOD, CAT, GPx, glutathione } \\
\text { reductase, GSH, GCLC, and GCLM }\end{array}$ & [19] \\
\hline
\end{tabular}

Abbreviations: ANP, atrial natriuretic peptide; $\alpha$-SMA, alpha-smooth muscle actin; Bax, Bcl-2-associated X protein; Bcl-2, B-cell lymphoma 2; BNP, brain natriuretic peptide; CAT, catalase; CAVI, caveolin-I; CTGF, connective tissue growth factor; GCLC, glutamate-cysteine ligase catalytic subunit; GCLM, glutamate cysteine ligase modifier; GPx, glutathione peroxidase; GSH, reduced glutathione; HO-I, heme oxygenase-I; IL-6, interleukin-6; Keap-I, Kelch-like ECH-associated protein I; LPS, lipopolysaccharides; NOX2, NADPH oxidases 2; NOX4, NADPH oxidases 4; NQOI, NADPH dehydrogenase quinone I; Nrf-2, nuclear factor erythroid 2-related factor; p47 ${ }^{\text {hox }}$ and p67 ${ }^{\text {phox }}$,

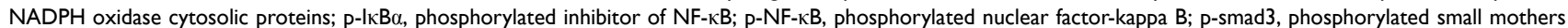
against decapentaplegic 3 ; ROS, reactive oxygen species; SOD, superoxide dismutase; TGF- $\beta$, transforming growth factor-beta; TNF- $\alpha$, tumour necrosis factor-alpha.

In autoimmune myocarditis (an inflammation of myocardium induced by autoimmune disease), the untreated rats had higher numbers of positive $\mathrm{CD}^{+}$cells, $\mathrm{CD} 14^{+}$cells and inflammatory cells in the myocardial tissue. Plasma inflammatory cytokines and anti-myosin antibody were increased, but interleukin-10 (IL-10) was decreased without treatment of andrographolide. The induction of inflammatory response resulted from the activation of PI3K and its downstream molecule, Akt. All these alterations during autoimmune myocarditis were reversed in the andrographolide-treated group. ${ }^{43}$ Andrographolide shifted the macrophage phenotype from proinflammatory subset $\left(\mathrm{CD}^{+} 6^{+}\right.$cell number was decreased) to anti-inflammatory subset (CD206 ${ }^{+}$cell number was increased) in mice fed by high-fat diet displaying cardiac injury, subsequently lowered the serum concentration of tumour necrosis factor-alpha (TNF- $\alpha$ ), monocyte chemoattractant protein-1 (MCP-1), high sensitivity C-reactive protein (hs-CRP) and interleukin-1 beta (IL-1 $\beta$ ). The suppression of inflammatory response was mediated through the inhibition of NF-kB. ${ }^{46}$ In diabetic cardiomyopathy, andrographolide blocked NF$\kappa \mathrm{B}-$ mediated inflammatory response. As a result, the protein expression of cyclooxygenase-2 (COX-2), TNF- $\alpha$, IL-1 $\beta$, interleukin-6 (IL-6), intercellular cell adhesion molecules-1 (ICAM-1), vascular cell adhesion molecule-1 (VCAM-1) were hindered. These findings were supported in vitro using H9C2 cardiomyocytes stimulated by high concentration of glucose. Andrographolide treatment suppressed high glucoseinduced I $\kappa \mathrm{B} \alpha$ phosphorylation and subsequent active NF- $\kappa \mathrm{B}$ nuclear translocation. ${ }^{44}$ In LPS-induced cardiac malfunctions, Zhang et al proved that the administration of LPS caused phosphorylation of $\mathrm{I} \kappa \mathrm{B} \alpha$ and overwhelming production of cytokines, which were attenuated by oral administration of andrographolide. ${ }^{42}$ These findings were in line with in vitro study by Feng et al (2017), describing that the incubation of andrographolide lowered TNF- $\alpha$ and IL-6 in LPS-stimulated primary rat myocardial microvascular endothelial cells. ${ }^{59}$ In addition, andrographolide protected against adverse cardiac remodelling through the mitigation of inflammation. The dephosphorylation of NF- $\kappa \mathrm{B}$, reduction of cytokines expression, $\mathrm{CD}_{4} 5^{+}$cells, and $\mathrm{CD} 8^{+}$cells were noted in the andrographolide-treated mice with myocardial infarction induced by left coronary artery ligation. ${ }^{18}$ The mechanisms of action of A. paniculata (Burm.f.) Nees and andrographolide in alleviating inflammatory response have been depicted in Figure 3.

\section{Regulation of Oxidative Stress}

Oxidative and nitrosative stress have been proposed as the molecular events in myocardial injury. It is the consequence of an imbalance between production and elimination of reactive oxygen species (ROS) and reactive nitrogen species (RNS) and/or depletion of antioxidant defence system. Superoxide anion $\left(\mathrm{O}_{2}{ }^{-}\right)$, hydroxyl radical 


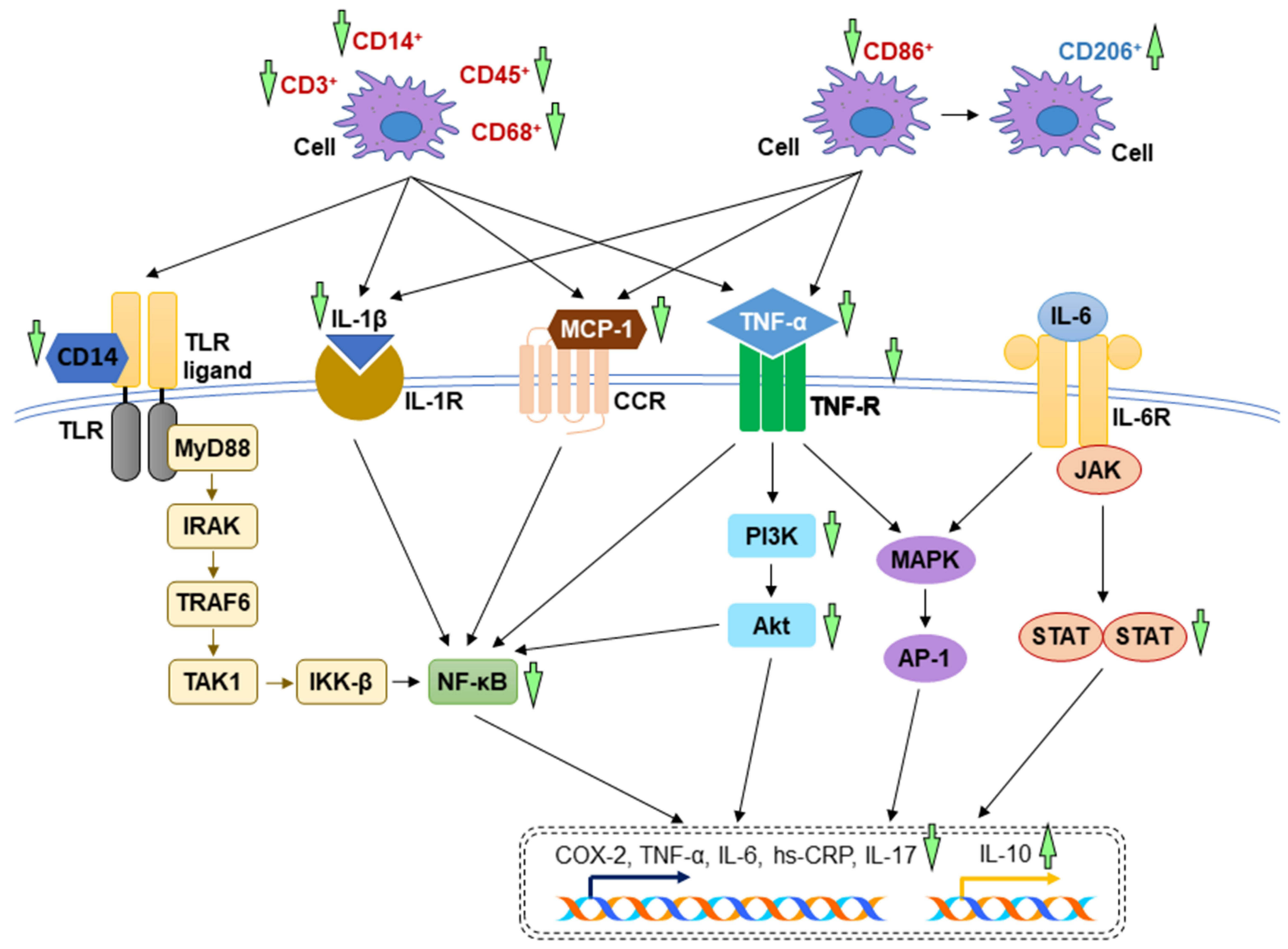

Figure 3 The mechanism of action of in the regulation of inflammatory response. During myocardial injuries, the overwhelming inflammatory cytokines activate several essential signalling pathways including TLR, NF-kB, PI3K/Akt, MAPK, JAK-STAT signal transduction pathways. The reductions of inflammation by A. paniculata (Burm.f.) Nees and andrographolide are mainly mediated through the inhibition of these signalling pathways (indicated by green arrows). The arrow pointing upward indicates an increase or activation while the arrow pointing downward indicates a decrease or inhibition.

$(\mathrm{OH} \bullet)$, hydrogen peroxide $\left(\mathrm{H}_{2} \mathrm{O}_{2}\right)$, nitric oxide $(\mathrm{NO} \bullet)$, and peroxynitrite $\left(\mathrm{ONOO}^{-}\right)$are the most common free radicals produced as metabolic by-products. ${ }^{60}$ Myocardial injury activates ROS-generating nicotinamide adenine dinucleotide phosphate (NADPH) oxidases, xanthine oxidase, cyclooxygenase, lipoxygenase, myeloperoxidase, and RNS-generating inducible nitric oxide synthase (iNOS) directly or indirectly via increased inflammatory response. ${ }^{61}$ The production of $\mathrm{O}_{2}{ }^{-}$coupled with $\mathrm{NO} \bullet$ will yield $\mathrm{ONOO}^{-}$. Inadequate antioxidant capacity with low levels of superoxide dismutase (SOD), glutathione peroxidase (GPx), catalase (CAT), and reduced glutathione (GSH) during myocardial injury further exacerbate oxidative stress and result in lipid peroxidation, protein modification and deoxyribonucleic acid (DNA) damage. Nuclear factor erythroid 2-related factor (Nrf-2) is an important transcription factor for the regulation of oxidative stress.
Nrf-2 binds to Kelch-like ECH-associated protein 1 (Keap-1) and sequesters in the cytoplasm under physiological condition, promoting its ubiquitination and degradation. Under oxidative condition, Keap-1 interacts with ROS, thus releasing Nrf-2. Subsequently, Nrf-2 translocates into the nucleus, forms a complex with small musculoaponeurotic fibrosarcoma oncogene homolog (Maf) protein and binds to the antioxidant response element (ARE) to stimulate downstream transcription of heme oxygenase-1 (HO-1), NADPH dehydrogenase quinone 1 (NQO1), glutamate-cysteine ligase catalytic subunit (GCLC), glutathione-S-transferase (GST), SOD, CAT, and $\mathrm{GSH}^{62}$

The beneficial effects of $A$. paniculata (Burm.f.) Nees against oxidative stress have been proven in a canine model of left anterior descending coronary artery ligationinduced ischaemia and reperfusion injury. The myocardial 


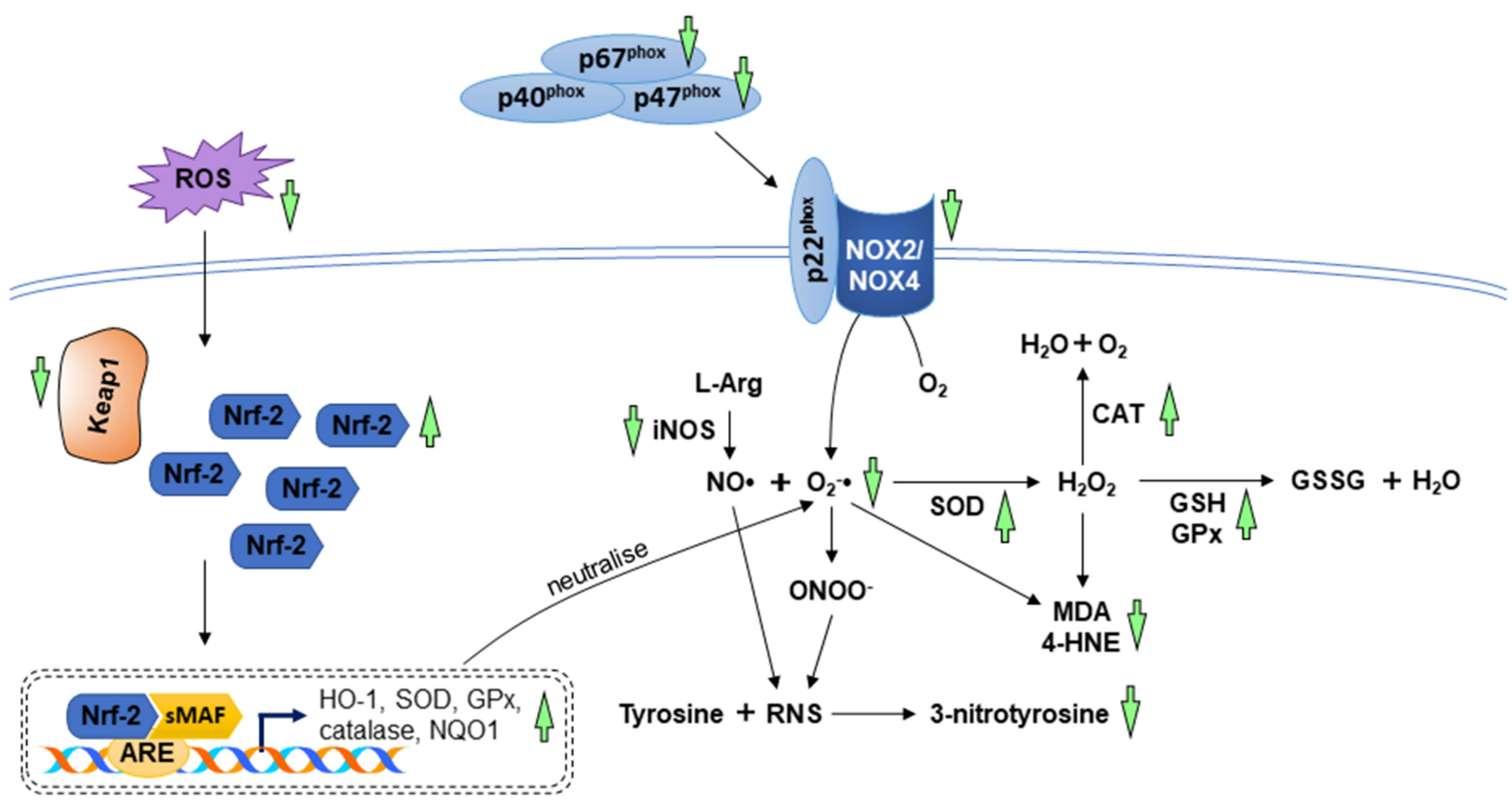

Figure 4 The mechanism of action in the regulation of oxidative stress. During myocardial injuries, the imbalance between ROS/RNS levels and anti-oxidative capacity cause lipid peroxidation and protein modification. The anti-oxidative property A. paniculata (Burm.f.) Nees and andrographolide are accomplished by enhancing the antioxidant system via activation of Nrf-2/Keap-I pathway as well as decreasing the enzymes responsible for oxidative and nitrosative stress (indicated by green arrows). The arrow pointing upward indicates an increase or activation while the arrow pointing downward indicates a decrease or inhibition.

tissues of mongrel dogs showed an increase in SOD activity, intracellular calcium, and a decrease in malondialdehyde (MDA) level after treatment with A. paniculata (Burm.f.) Nees. ${ }^{37,38}$ Ojha and co-researchers reported restoration of antioxidant capacity and inhibition of lipid peroxidation after treatment of $A$. paniculata (Burm.f.) Nees in two experimental rat models of ischaemia and reperfusion injury induced by left anterior descending coronary artery ligation as well as myocardial injury induced by isoproterenol. ${ }^{40,41}$

Several reports revealed the beneficial effects of andrographolide against oxidative stress in animals and cardiomyocytes. It has been reported that andrographolide suppressed the level of oxidative stress biomarkers (MDA, 4-hydroxynonenal, 3-nitrotyrosine, and ROS) in myocardial dysfunction induced by diabetes and left coronary artery ligation. The anti-oxidative action of andrographolide was achieved by inhibiting the enzymes responsible for ROS and RNS synthesis as well as targeting the Nrf-2 signalling axis known for the enhancement of antioxidant activities. Andrographolide inhibited the expression of iNOS, NADPH oxidases 2 (NOX2), NADPH oxidase 4 (NOX4), and NADPH oxidase cytosolic proteins ( $\mathrm{p} 47^{\text {phox }}$ and $\left.\mathrm{p} 67^{\mathrm{phox}}\right)$. Additionally, andrographolide enhanced Nrf-2 nuclear translocation and upregulated downstream expression of HO-1, NQO1, SOD, and GPx in the heart. ${ }^{18,44}$ Cardiac NO• levels enhanced by LPS injection was reversed by andrographolide in male mice. ${ }^{42}$ Results from in vitro studies demonstrated similar outcomes, in which Nrf-2 nuclear translocation was promoted, whereas NADPH oxidases and their cytosolic proteins were suppressed in cardiomyocytes subjected to high concentration of glucose or hypoxia and treated with andrographolide. ${ }^{18,44}$ Another cell culture study depicted that andrographolide treatment protected cardiomyocytes against hypoxia and reoxygenation injury by upregulating enzymatic antioxidant activities, non-enzymatic antioxidant content, GCLC and glutamate cysteine ligase modifier (GCLM) expression. ${ }^{19}$ The anti-oxidative actions of $A$. paniculata (Burm.f.) Nees and andrographolide have been summarised in Figure 4.

\section{Regulation of Apoptosis}

Apoptosis is a process of programmed cell death, which has a pathological implication in myocardial loss, resulted from the loss of mitochondrial and endoplasmic reticulum mass leading to disruption of cellular integrity. ${ }^{63,64}$ Apoptosis is tightly regulated by the extrinsic and intrinsic mechanism in response to the exogenous and endogenous triggering stimulus, respectively. The extrinsic mechanism 


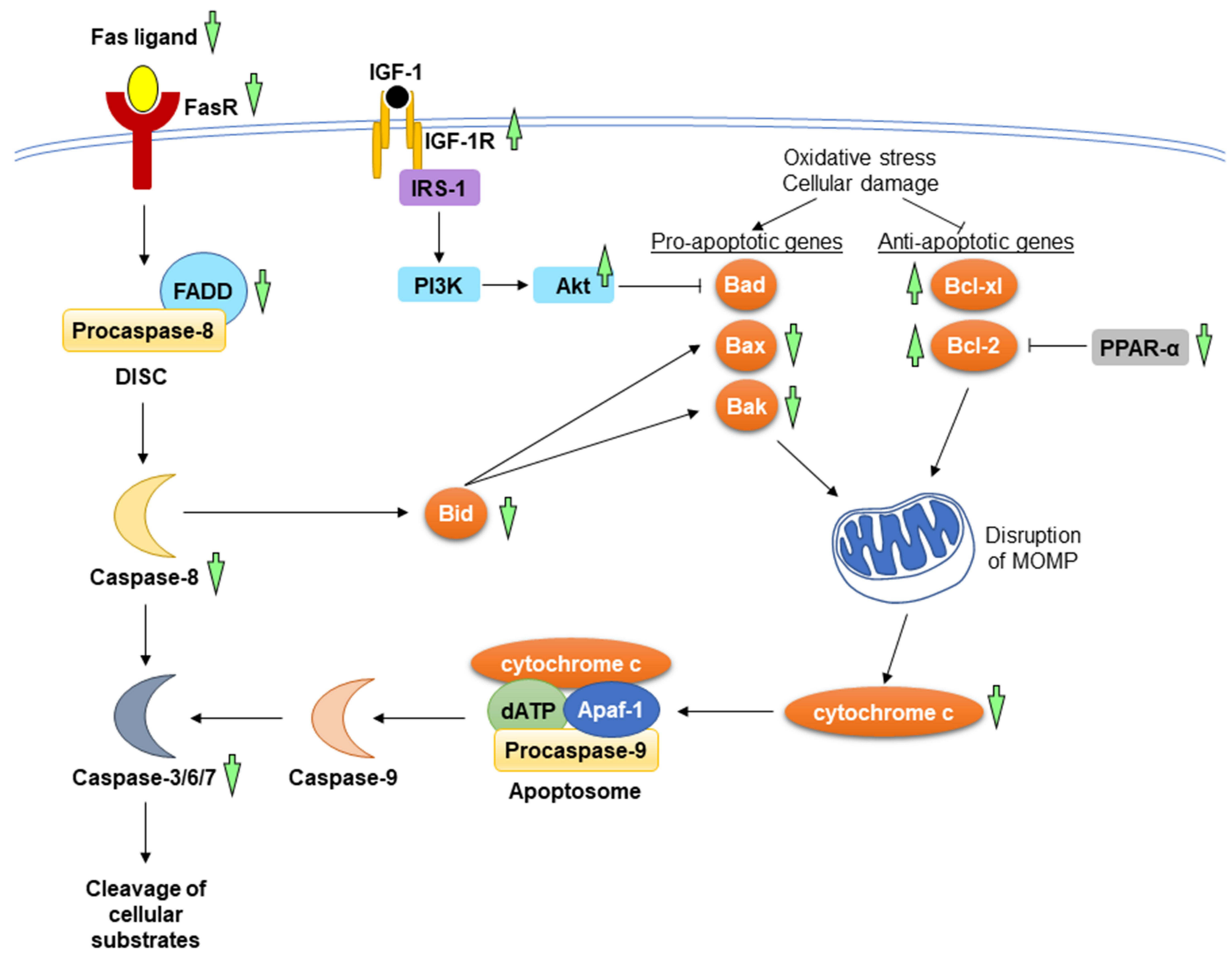

Figure 5 The mechanism of action in the regulation of apoptosis during myocardial injuries. In the extrinsic pathway, the interaction between Fas ligand and its receptor causes the recruitment of FADD and procaspase-8 to form DISC. In the intrinsic pathway, the increases in pro-apoptotic genes and the decrease in anti-apoptotic genes resulted from oxidative stress and cellular damage lead to the release of cytochrome $c$ from mitochondria. Apoptosome formation ensues, consisting of cytochrome $c$, deoxyadenosine triphosphate (dATP), apoptotic protease-activating factor I (Apaf-I), and procaspase-9. The activated initiator caspases (caspase-8 and caspase-9) further activates executioner caspases (caspase-3, caspase-6, and caspase-7), promoting the cleavage of cellular substrate. A. paniculata (Burm.f.) Nees and andrographolide prevent apoptosis by suppressing the mitochondrial and death receptor pathways. The upstream signalling events involved are the activation of IGF-IR and inhibition of PPAR- $\alpha$. The anti-apoptotic effects of $A$. paniculata (Burm.f.) Nees and andrographolide are indicated (green arrows). The arrow pointing upward indicates an increase or activation while the arrow pointing downward indicates a decrease or inhibition.

(also known as death receptor pathway) is initiated upon ligation of Fas ligand and tumour necrosis factor-related apoptosis-inducing ligand (TRAIL) with their receptors, Fas and TRAIL receptor. Formation of death-inducing signalling complex (DISC) ensues, comprising Fasassociated death domain protein (FADD) and initiator caspase-8. The intrinsic mitochondrial pathway is promoted in the presence of cellular damage, hypoxia, and survival factor deprivation. These intracellular signals alter the expression of B-cell lymphoma 2 (Bcl-2), B-cell lymphoma-extra large (Bcl-xl), Bcl-2-associated $\mathrm{X}$ protein (Bax) and Bcl-2-antagonist/killer (Bak), whereby the former two genes favour apoptosis inhibition, while the latter two genes favour apoptosis activation. Raised proapoptotic genes and lowered anti-apoptotic genes enhance the release of cytochrome c, leading to apoptosome formation and initiator caspase- 9 activation. The activated initiator caspases from both pathways, in turn, activate executioner caspases (caspase-3, caspase-6, and caspase7) essential for the cleavage of cellular substrates, such as cytokeratins, cytoskeletal, and poly (ADP-ribose) polymerase (PARP). ${ }^{65,66}$ The two different apoptosis pathways can be bridged by a pro-apoptotic BH3-interacting domain (Bid), which is cleaved by activated caspase- 8 upon death receptor stimulation. The translocation of Bid into mitochondria leads to the release of cytochrome c. ${ }^{67}$ Insulin- 
like growth factor-1 (IGF-1) and peroxisome proliferatoractivated receptor-alpha (PPAR- $\alpha$ ) are pivotal signalling molecules in apoptosis regulation. Interaction between IGF-1 and its receptor (IGF-1R) causes autophosphorylation at $\beta$-subunits of the receptor, which provides a docking site for insulin receptor substrate-1 (IRS-1). This eventually drives the phosphorylation and activation of PI3K and its downstream protein kinase, Akt. The activated Akt, in turn, inactivates Bcl-2-associated agonist of cell death (Bad), thus inhibiting apoptosis. ${ }^{68}$ On the other hand, PPAR- $\alpha$ promotes Bcl-2 ubiquitination and degradation, resulting in cell apoptosis. ${ }^{69}$

A. paniculata (Burm.f.) Nees and andrographolide potentially provide cardioprotection via mitigation of apoptosis event, indicated by the reduction of terminal deoxynucleotidyl transferase dUTP nick end labelling (TUNEL)-positive cardiac cells and cleaved PARP activity. ${ }^{17,44,45}$ A. paniculata (Burm.f.) Nees extract attenuated pathological cardiac hypertrophy governed through its apoptosis-suppressing properties in both extrinsic and intrinsic pathways. Feeding the animals with high-fat diet increased the protein levels of Fas ligand, Fas receptor, FADD, Bid, caspase-8, and caspase 3, in which these apoptotic proteins were decreased after treated with A. paniculata (Burm.f.) Nees extract. Besides, the proapoptotic proteins (Bad and Bak) and cytochrome c accumulation were lowered in the A. paniculata (Burm. f.) Nees-treated obese mice. ${ }^{17}$

Andrographolide exerted anti-apoptotic effects by orchestrating both extrinsic and intrinsic pathways. The expression of Fas receptor and FADD in mice fed with high-fat diet was suppressed by andrographolide. ${ }^{45}$ For the intrinsic pathway, the protein levels of Bcl-2 and Bcl-xl were upregulated, whereas Bax, Bak, cytochrome $\mathrm{c}$, and caspase-3 were downregulated in high-fat diet-induced obese mice following the administration of andrographolide. Concomitantly, the protein level of phosphorylated IGF-1R was increased, while the expression of PPAR- $\alpha$ was downregulated after being treated with andrographolide. ${ }^{45,46}$ A comprehensive study consisting of in vivo and in vitro experiments found reductions of Bax/Bcl-2 ratio and caspase- 3 activity in the andrographolide-treated diabetic mice with myocardial dysfunction and in $\mathrm{H} 9 \mathrm{C} 2$ cardiomyocytes stimulated by high concentration of glucose. The authors suggested that the anti-apoptotic activity of andrographolide was mediated through activation of Akt activity. ${ }^{44}$ Another study showed that andrographolide suppressed LPS-induced myocardial apoptosis as well as inhibited the executioner caspase- 3 and caspase7 activities for in mouse heart. ${ }^{42}$ The molecular mechanisms underlying the anti-apoptotic effects of $A$. paniculata (Burm.f.) Nees and andrographolide have been summarised in Figure 5.

\section{Prevention of Cardiac Fibrosis and Endothelial Dysfunction}

Cardiac fibrosis is defined as an imbalance between the production and degradation of extracellular matrix (ECM), leading to scar tissue accumulation, heart architecture distortion, and cardiac dysfunction. ${ }^{70}$ During myocardial injury, the pathogenesis of cardiac fibrosis is initiated with the increases in numerous profibrotic growth factors, such as transforming growth factor-beta (TGF- $\beta$ ), connective tissue growth factor (CTGF), and endothelin-1 (ET-1). TGF- $\beta$ is a pleiotropic regulator in cardiac fibrosis. It stimulates the conversion of cardiac fibroblasts into myofibroblasts, promotes collagen deposition by decreasing the activity of plasminogen activators [such as urokinase plasminogen activator (u-PA) and tissue plasminogen activator (t-PA)] as well as inhibiting ECM degradation by decreasing matrix metalloproteinases (MMPs) activation via stimulation of plasminogen activator inhibitor-1 (PAI1). ${ }^{71}$ In addition to the role as mediators for collagen synthesis and ECM degradation, plasminogen activators are proteins that facilitate the conversion of plasminogen to plasmin (an enzyme involved in the physiological breakdown of blood clot), whereas PAI-1 is a principal inhibitor of plasminogen activators, thus suggesting their functions in fibrinolytic activity. The expression of CTGF in myocardium is highly induced by cytokines and it creates a fibrotic environment and modulates the activity of other growth factors in ECM. Endothelin-1 promotes cardiac fibroblast proliferation, collagen production, and microfibroblast differentiation. Apart from the role of ET-1 in regulating cardiac fibrosis, it is also a powerful endogenous vasoconstrictor produced predominantly by endothelial cells. Its action is opposed to the function of $\mathrm{NO} \cdot$, whereby ET-1 exerted an inhibitory effect on NO•mediated vasodilatation. Hence, the changes in $\mathrm{NO} \cdot$ and ET-1 cause vascular endothelial dysfunction. ${ }^{72}$ Endothelial dysfunction is characterised by the reduction in bioavailability of $\mathrm{NO} \bullet$ (a vasodilator) and/or an imbalance between endothelium-derived contracting and relaxing factors. Endothelial dysfunction precedes the development of myocardial injury. Mechanistically, these profibrotic growth 


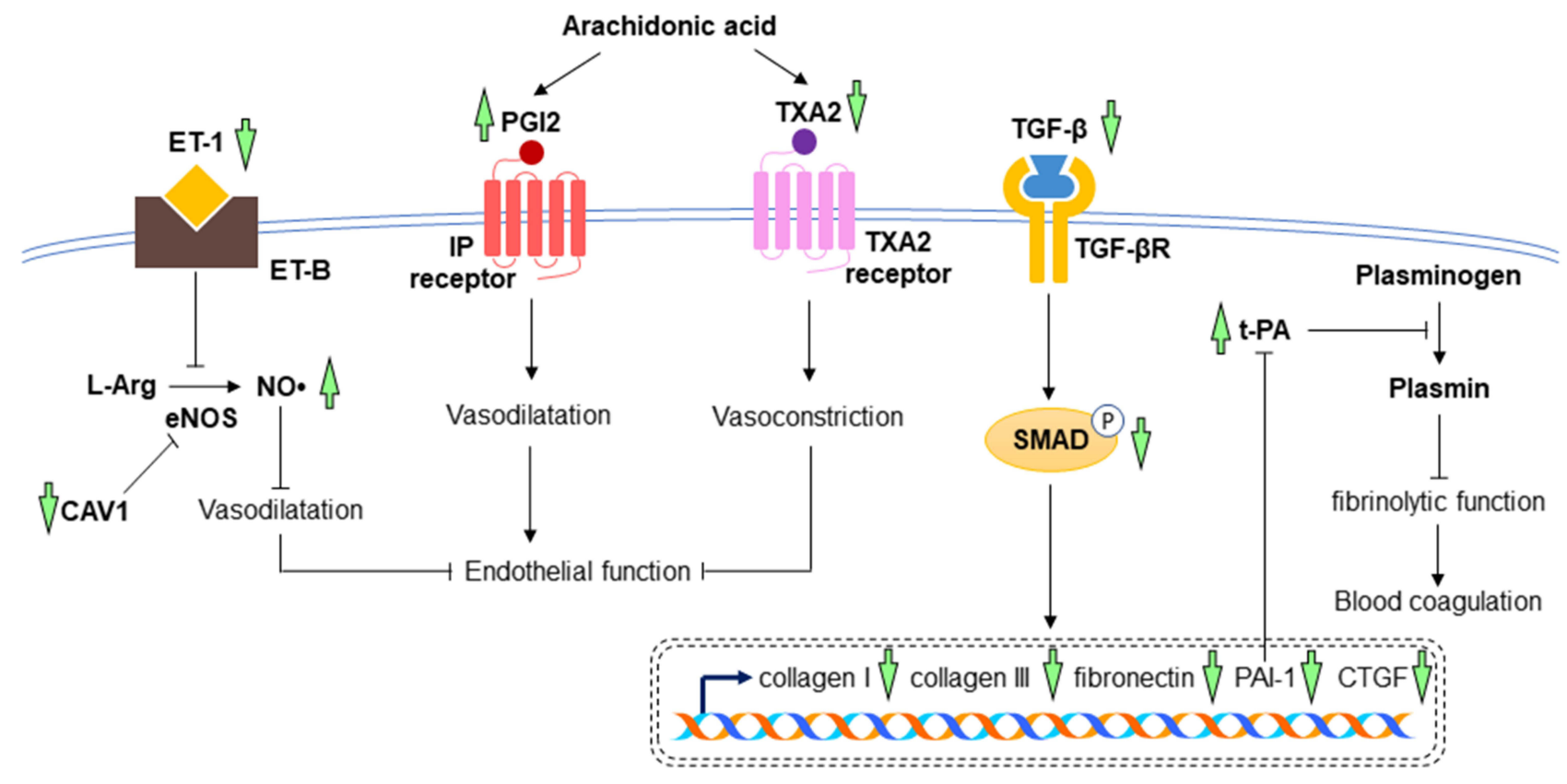

Figure 6 The mechanism of action in the prevention of cardiac fibrosis and endothelial dysfunction. In myocardial injuries, the increased expressions of collagen I, collagen III, fibronectin, PAI-I, and CTGF are mediated through TGF- $\beta / S M A D$ signalling pathway, leading to the increase in blood coagulation and cardiac fibrosis. High levels of ET-I, TXA2 and low level of PGI2 cause the inhibition of vasodilatation, resulting in endothelial dysfunction. Treatment with A. paniculata (Burm.f.) Nees and andrographolide reverse all these changes, leading to the reduction of cardiac fibrosis, improvement of endothelial function and fibrinolytic function (indicated by green arrows). The arrow pointing upward indicates an increase or activation while the arrow pointing downward indicates a decrease or inhibition.

factors trigger small mothers against decapentaplegic (Smad)-dependent and Smad-independent pathways to regulate ECM homeostasis. All the profibrotic growth factors are positively correlated with each other, further providing a positive feedback loop on fibrotic response. ${ }^{70}$ Thromboxane A2 (TXA2) is a potent vasoconstrictor and has a prothrombotic property. It is produced by activated platelet to promote platelet aggregation. Nonetheless, prostacyclin or prostaglandin I2 (PGI2) is an effective vasodilator produced by vascular endothelium and has an inhibitory effect on platelet activation. Therefore, changes in TXA2 and PGI2 affect endothelial function and they work as physiological antagonists for the regulation of blood coagulation in the vascular system. In the endothelial cells, caveolin-1 (CAV1) binds to endothelial nitric oxide synthase (eNOS) and inhibits NO• production. ${ }^{73}$

The promising effects of $A$. paniculata (Burm.f.) Nees against ischemic myocardium in experimental dogs were also mediated through its fibrinolytic action. The levels of PGI2 in platelets and euglobulin lysis time (a measure of fibrinolysis) were elevated, whereas the level of TXA2 was lowered in the $A$. paniculata (Burm.f.) Nees-treated group. ${ }^{39}$ Andrographolide has been proven to alleviate cardiac fibrosis through the TGF- $\beta /$ Smad pathway. The collagen deposition and expression of fibrosis markers, including collagen I, collagen III, TGF- $\beta$, and fibronectin, were markedly reduced by andrographolide in streptozotocin-induced diabetic mice as compared to non-treated negative controls. ${ }^{44}$ In recent year, Xie et al performed preclinical studies utilising a mouse model of myocardial infarction induced by left coronary artery ligation and hypoxia-induced cardiomyocytes. The results showed the increases in TGF- $\beta$, collagen I, collagen III, CTGF, and phosphorylated Smad caused by myocardial infarction or hypoxia were attenuated by andrographolide treatment. ${ }^{18}$ Andrographolide also mitigated vascular endothelial dysfunction caused by chronic heart disease by decreasing ET-1 and TXA2 while increasing the levels of NO• and PGI2. Besides, andrographolide elevated t-PA and reduced PAI-1 content, indicating the improvement of fibrinolytic function and reduction of blood coagulation in mice with chronic heart disease. ${ }^{46}$ Microarray analysis also showed a reduction in CAV1 by andrographolide, which might help in maintaining $\mathrm{NO} \cdot$ level and improved endothelial function in LPS-stimulated primary rat myocardial microvascular endothelial cells. ${ }^{59}$ The postulated mechanisms of A. paniculata (Burm.f.) Nees and andrographolide in the prevention of cardiac fibrosis and endothelial dysfunction have been summarised in Figure 6. 
Taken together, the evidence showed that $A$. paniculata (Burm.f.) Nees and andrographolide potentially mitigate myocardial injury in animal and cellular models via its anti-inflammatory, anti-oxidative, and anti-apoptotic properties. The inhibition of important signalling molecules (such as NF-kB, PI3K/Akt, and MAPK) is responsible for regulating the crosstalk between myocardial injury and inflammation. The reduction of oxidative stress by A. paniculata (Burm.f.) Nees and andrographolide is in part modulated through the activation of Nrf-2 signalling axis. Both extrinsic and intrinsic pathways are involved in anti-apoptotic effects of $A$. paniculata (Burm.f.) Nees and andrographolide in heart tissues. Moreover, $A$. paniculata (Burm.f.) Nees and andrographolide prevents cardiac fibrosis, endothelial dysfunction, and enhances fibrinolytic activity, conferring cardioprotective effects for the prevention of myocardial injury.

\section{Safety and Toxicity of Andrographolide}

A wide array of cellular, animal, and human studies revealed that $A$. paniculata (Burm.f.) Nees and andrographolide are safe and non-toxic. There was no observable adverse effect reported in rats even at a relatively high dose of andrographolide or A. paniculata (Burm.f.) Nees (up to $5 \mathrm{~g} / \mathrm{kg}$ ). ${ }^{30,74,75}$ The cytotoxic effect of andrographolide was not seen in primary rat myocardial microvascular endothelial cells at the dose of $10 \mu \mathrm{g} / \mathrm{mL} .{ }^{59}$ Batran et al evaluated the acute toxicity of andrographolide (100 or $500 \mathrm{mg} / \mathrm{kg}$ ) in healthy male and female rats by observing the behavioural changes, abnormal manifestation, and mortality rate up to 24-hour post-treatment. There were no death or hazardous signs in biochemical parameters and histological evaluation in renal, liver, and kidney after andrographolide administration. ${ }^{76}$ In humans, the subjects were well tolerated with the doses of $A$. paniculata (Burm. f.) Nees (3 or $6 \mathrm{~g} / \mathrm{kg}$ ) provided and it is effective in relieving fever and sore throat in a randomised doubleblind study recruiting patients with pharyngotonsillitis. ${ }^{77}$ A Phase I trial of andrographolide from A. paniculata (Burm.f.) Nees was conducted on human immunodeficiency virus-positive patients and healthy individuals. The regimen used was the dose escalation of andrographolide at the doses of 5,10 , and $20 \mathrm{mg} / \mathrm{kg}$ over a 9-week period ( 3 weeks for each dose). No adverse medical outcomes were observed, whereby the levels of aspartate aminotransferase (AST) and alanine aminotransferase
(ALT) were in the normal range throughout the study period. ${ }^{78}$ In brief, the scientific data obtained from preclinical and clinical settings assure the safety and medicinal use of A. paniculata (Burm.f.) Nees and andrographolide, which requires further validation in patients with myocardial injury.

\section{Perspectives}

The pharmacokinetics of A. paniculata (Burm.f.) Nees or andrographolide serve as the essential information for determining their optimum treatment dose, route, frequency, and duration for potential clinical utility. The absorption of A. paniculata (Burm.f.) Nees is almost complete following its oral administration. Plasma concentration of andrographolide reaches the peak at two hours after oral administration of $A$. paniculata (Burm.f.) Nees at the dose of 20 and $200 \mathrm{mg} / \mathrm{kg}^{79}$ In terms of distribution, andrographolide can be detected in the kidney, liver, heart, spleen, lung, and brain of rats after orally treated with A. paniculata (Burm.f.) Nees extract $(133.33 \mathrm{mg} / \mathrm{kg}$ ) and andrographolide $(100 \mathrm{mg} / \mathrm{kg})$, with the maximum concentration of andrographolide in these organs was observed at one-hour post-administration. ${ }^{80}$ The half-life of andrographolide was approximately 2.5 hours and excreted within 8 hours. ${ }^{79,80}$

As mentioned earlier, the effective doses of andrographolide against myocardial injury ranged from 10 to $100 \mathrm{mg} / \mathrm{kg}$ in animals. The calculated human equivalent dose is estimated to be $48-1000 \mathrm{mg}$ for a $60 \mathrm{~kg}$ adult, which can be used as dose reference for future human trials. However, it is worthy to note that treatment with the lowest dose of andrographolide for a week might exert lesser beneficial effects. For instance, significant changes were not observed in the levels of thiobarbituric acid reactive substance and SOD in male mice with LPS-induced cardiac malfunction. ${ }^{42}$ In addition, higher dosage of $A$. paniculata (Burm.f.) Nees $(100-2000 \mathrm{mg} / \mathrm{kg})$ than andrographolide might be required, possibly attributed to a lower amount of andrographolide or the presence of other phytoconstituents in the plant extract as compared to the pure compound. Likewise, a shorter period of treatment (one week) for A. paniculata (Burm.f.) Nees was also insufficient to induce changes in some apoptosis parameters in obese mice fed with high-fat diet. ${ }^{17}$ In this context, the use of andrographolide at lower dose can be considered over the use of A. paniculata (Burm.f.) Nees extract at higher dose if comparable protective action against myocardial injury can be achieved. Another possible factor influencing the effective 
dose of $A$. paniculata (Burm.f.) Nees is the quality of plant extract, whereby andrographolide content may vary geographically, environmentally, and seasonally. ${ }^{81,82}$ The evaluation of plant extracts and andrographolide content present in different extracts should be mentioned along with the geographical distribution. Thus, pure compound is preferable compared to its original plant extract for better estimation of effective dose.

To our knowledge, there is a lack of clinical trial assessing the effect of $A$. paniculata (Burm.f.) Nees and andrographolide in patients with myocardial injury. The absence of data on the comparison between the efficacy of A. paniculata (Burm.f.) Nees or andrographolide with standard therapy is the shortcoming of current preclinical evidence. It will be rather challenging to translate the findings clinically. However, the combination of A. paniculata (Burm.f.) Nees or andrographolide with standard therapy may be possible in future human trials.

\section{Conclusion}

The abilities of $A$. paniculata (Burm.f.) Nees and andrographolide in resolving overwhelming inflammatory response, oxidative stress, apoptosis event, cardiac fibrosis, and endothelial dysfunction contribute to their protective effects against myocardial injury. From the evidence gathered, it would appear that both A. paniculata (Burm.f.) Nees and andrographolide may have the potential to be developed as prophylactic agents against future myocardial injury. They appear to be less effective for short-term relief of myocardial events. There are also no obvious differences in the myocardial protective effects between A. paniculata (Burm.f.) Nees and andrographolide. However, the effective dose of A. paniculata (Burm.f.) Nees is generally higher than andrographolide. Thus, andrographolide may be a better choice to consider whereby a lower dose may be sufficient. To confirm whether andrographolide is the specific bioactive compound responsible for beneficial properties of A. paniculata (Burm.f.) Nees, both plant extract and andrographolide isolated from the plant should be tested in vitro or in vivo under the same experimental setting to compare their myocardial protective effects. Researchers should further evaluate the effects of $A$. paniculata (Burm.f.) Nees and andrographolide in clinical trials to verify the positive outcomes reported in vivo and in vitro, thus promoting their potential clinical application as the management for myocardial injury.

\section{Acknowledgment}

This study is supported by Universiti Kebangsaan Malaysia via FF-2020-366 grant.

\section{Disclosure}

The authors report no conflicts of interest in this work.

\section{References}

1. McCarthy CP, Raber I, Chapman AR, et al. Myocardial injury in the era of high-sensitivity cardiac troponin assays: a practical approach for clinicians. JAMA Cardiol. 2019;4:1034-1042. doi:10.1001/ jamacardio.2019.2724

2. DeFilippis AP, Chapman AR, Mills NL, et al. Assessment and treatment of patients with type 2 myocardial infarction and acute nonischemic myocardial injury. Circulation. 2019;140:1661-1678. doi:10.1161/CIRCULATIONAHA.119.040631

3. Kadesjö E, Roos A, Siddiqui Anwar J, et al. Treatment with cardiovascular medications: prognosis in patients with myocardial injury. $J$ Am Heart Assoc. 2021;10:e017239. doi:10.1161/JAHA.120.017239

4. Lu J, Ma Y, Wu J, et al. A review for the neuroprotective effects of andrographolide in the central nervous system. Biomed Pharmacother. 2019;117:109078. doi:10.1016/j.biopha.2019.109078

5. Khan I, Khan F, Farooqui A, et al. Andrographolide exhibits anticancer potential against human colon cancer cells by inducing cell cycle arrest and programmed cell death via augmentation of intracellular reactive oxygen species level. Nutr Cancer. 2018;70:787-803. doi:10.1080/01635581.2018.1470649

6. Pearngam P, Kumkate S, Okada S, et al. Andrographolide inhibits cholangiocarcinoma cell migration by down-regulation of claudin-1 via the p-38 signaling pathway. Front Pharmacol. 2019;10:827. doi:10.3389/fphar.2019.00827

7. Yue GG, Li L, Lee JK, et al. Multiple modulatory activities of Andrographis paniculata on immune responses and xenograft growth in esophageal cancer preclinical models. Phytomedicine. 2019;60:152886. doi:10.1016/j.phymed.2019.152886

8. Banerjee M, Parai D, Chattopadhyay S, et al. Andrographolide: antibacterial activity against common bacteria of human health concern and possible mechanism of action. Folia Microbiol. 2017;62:237-244.

9. Rasool U, P S, Parveen A, et al. Efficacy of Andrographis paniculata against extended spectrum $\beta$-lactamase (ESBL) producing E. coli. BMC Complement Altern Med. 2018;18:244. doi:10.1186/s12906018-2312-8

10. Hassan WRM, Basir R, Ali AH, et al. Anti-malarial and cytokine-modulating effects of andrographolide in a murine model of malarial infection. Trop Biomed. 2019;36:776-791.

11. Mishra K, Dash AP, Swain BK, et al. Anti-malarial activities of Andrographis paniculata and Hedyotis corymbosa extracts and their combination with curcumin. Malar J. 2009;8:26. doi:10.1186/14752875-8-26

12. Trivedi NP, Rawal UM, Patel BP. Hepatoprotective effect of andrographolide against hexachlorocyclohexane-induced oxidative injury. Integr Cancer Ther. 2007;6:271-280. doi:10.1177/ 1534735407305985

13. Chua LS. Review on liver inflammation and antiinflammatory activity of Andrographis paniculata for hepatoprotection. Phytother Res. 2014;28:1589-1598. doi:10.1002/ptr.5193

14. Cabrera D, Wree A, Povero D, et al. Andrographolide ameliorates inflammation and fibrogenesis and attenuates inflammasome activation in experimental non-alcoholic steatohepatitis. Sci Rep. 2017;7:3491. doi:10.1038/s41598-017-03675-z 
15. Mussard E, Jousselin S, Cesaro A, et al. Andrographis paniculata and its bioactive diterpenoids against inflammation and oxidative stress in keratinocytes. Antioxidants. 2020;9:530.

16. Duan MX, Zhou H, Wu QQ, et al. Andrographolide protects against HG-induced inflammation, apoptosis, migration, and impairment of angiogenesis via PI3K/AKT-eNOS signalling in HUVECs. Mediators Inflamm. 2019;2019:6168340. doi:10.1155/2019/6168340

17. Hsieh YL, Shibu MA, Lii CK, et al. Andrographis paniculata extract attenuates pathological cardiac hypertrophy and apoptosis in high-fat diet fed mice. J Ethnopharmacol. 2016;192:170-177. doi:10.1016/j. jep.2016.07.018

18. Xie S, Deng W, Chen J, et al. Andrographolide protects against adverse cardiac remodeling after myocardial infarction through enhancing Nrf2 signaling pathway. Int J Biol Sci. 2020;16:12-26. doi:10.7150/ijbs.37269

19. Woo AY, Waye MM, Tsui SK, et al. Andrographolide up-regulates cellular-reduced glutathione level and protects cardiomyocytes against hypoxia/reoxygenation injury. $J$ Pharmacol Exp Ther. 2008;325:226-235. doi:10.1124/jpet.107.133918

20. Hu XY, Wu RH, Logue M, et al. Andrographis paniculata (Chuān Xīn Lián) for symptomatic relief of acute respiratory tract infections in adults and children: a systematic review and meta-analysis. PLoS One. 2017;12:e0181780. doi:10.1371/journal.pone.0181780

21. Widjajakusuma EC, Jonosewojo A, Hendriati L, et al. Phytochemical screening and preliminary clinical trials of the aqueous extract mixture of Andrographis paniculata (Burm. f.) Wall. ex Nees and Syzygium polyanthum (Wight.) Walp leaves in metformin treated patients with type 2 diabetes. Phytomedicine. 2019;55:137-147. doi:10.1016/j.phymed.2018.07.002

22. Langhorst J, Wulfert $H$, Lauche $R$, et al. Systematic review of complementary and alternative medicine treatments in inflammatory bowel diseases. J Crohns Colitis. 2015;9:86-106. doi:10.1093/eccojcc/jju007

23. Bertoglio JC, Baumgartner M, Palma R, et al. Andrographis paniculata decreases fatigue in patients with relapsing-remitting multiple sclerosis: a 12-month double-blind placebo-controlled pilot study. BMC Neurol. 2016;16:77. doi:10.1186/s12883-016-0595-2

24. Hancke JL, Srivastav S, Cáceres DD, et al. A double-blind, randomized, placebo-controlled study to assess the efficacy of Andrographis paniculata standardized extract $\left(\right.$ ParActin $\left.^{\circledR}\right)$ on pain reduction in subjects with knee osteoarthritis. Phytother Res. 2019;33:1469-1479. doi:10.1002/ptr.6339

25. Cáceres DD, Hancke JL, Burgos RA, et al. Use of visual analogue scale measurements (VAS) to assess the effectiveness of standardized Andrographis paniculata extract SHA-10 in reducing the symptoms of common cold. A randomized double blind-placebo study. Phytomedicine. 1999;6:217-223. doi:10.1016/S0944-7113(99) 80012-9

26. Phunikhom K, Khampitak K, Aromdee C, et al. Effect of andrographis paniculata extract on triglyceride levels of the patients with hypertriglyceridemia: a randomized controlled trIAL. J Med Assoc Thai. 2015;98(Suppl 6):S41-47.

27. Ciampi E, Uribe-San-Martin R, Cárcamo C, et al. Efficacy of andrographolide in not active progressive multiple sclerosis: a prospective exploratory double-blind, parallel-group, randomized, placebo-controlled trial. BMC Neurol. 2020;20:173. doi:10.1186/ s12883-020-01745-w

28. Burgos RA, Hancke JL, Bertoglio JC, et al. Efficacy of an Andrographis paniculata composition for the relief of rheumatoid arthritis symptoms: a prospective randomized placebo-controlled trial. Clin Rheumatol. 2009;28:931-946. doi:10.1007/s10067-0091180-5

29. Li X, Zhang C, Shi Q, et al. Improving the efficacy of conventional therapy by adding andrographolide sulfonate in the treatment of severe hand, foot, and mouth disease: a randomized controlled trial. Evid Based Complement Alternat Med. 2013;2013:316250.
30. Bothiraja C, Pawar AP, Shende VS, et al. Acute and subacute toxicity study of andrographolide bioactive in rodents: evidence for the medicinal use as an alternative medicine. Comp Clin Path. 2013;22:1123-1128. doi:10.1007/s00580-012-1539-X

31. Allan JJ, Pore MP, Deepak M, et al. Reproductive and fertility effects of an extract of Andrographis paniculata in male Wistar rats. Int J Toxicol. 2009;28:308-317. doi:10.1177/1091581809339631

32. Rajani M, Shrivastava N, Ravishankara MN. A rapid method for isolation of andrographolide from andrographis paniculata nees (kalmegh). Pharm Biol. 2000;38:204-209. doi:10.1076/1388-0209(200007)3831-SFT204

33. Pundarikakshudu K, Shah PA, Panchal SR, et al. A simple and facile method for the isolation of andrographolide from andrographis paniculata nees; 2016

34. Villedieu-Percheron E, Ferreira V, Campos JF, et al. Quantitative determination of andrographolide and related compounds in andrographis paniculata extracts and biological evaluation of their anti-inflammatory activity. Foods. 2019;8:683.

35. Rivera D, Allkin R, Obón C, et al. What is in a name? The need for accurate scientific nomenclature for plants. J Ethnopharmacol. 2014;152:393-402. doi:10.1016/j.jep.2013.12.022

36. Zhiling G, Huayue Z, Liangwu L. Protective effects of API0134 on myocardial ischemia and reperfusion injury. J Tongji Med Univ. 1996;16:193-197. doi:10.1007/BF02888103

37. Zhi-ling G, Hua-yue Z, Xin-hua Z. The effect of andrographis paniculata nees (APN) in alleviating the myocardial ischemic reperfusion injury. J Tongji Med Univ. 1994;14:49-51. doi:10.1007/BF02888059

38. Zhi-ling G, Hua-yue Z, Xin-hua Z. An experimental study of the mechanism of andrographis paniculata nees (APN) in alleviating the $\mathrm{Ca}(2+)$-overloading in the process of myocardial ischemic reperfusion. J Tongji Med Univ. 1995;15:205-208. doi:10.1007/ BF02887945

39. Hua-yue Z, Wei-yi F. Protective effects of Andrographis paniculata Nees on post-infarction myocardium in experimental dogs. $J$ Tongji Med Univ. 1990;10:212-217. doi:10.1007/BF02887933

40. Ojha S, Bharti S, Golechha M, et al. Andrographis paniculata extract protect against isoproterenol-induced myocardial injury by mitigating cardiac dysfunction and oxidative injury in rats. Acta Pol Pharm. 2012;69:269-278.

41. Ojha SK, Bharti S, Joshi S, et al. Protective effect of hydroalcoholic extract of Andrographis paniculata on ischaemia-reperfusion induced myocardial injury in rats. Indian J Med Res. 2012;135:414-421.

42. Zhang J, Zhu D, Wang Y, et al. Andrographolide attenuates LPSinduced cardiac malfunctions through inhibition of IкB phosphorylation and apoptosis in mice. Cell Physiol Biochem. 2015;37:1619-1628. doi:10.1159/000438528

43. Zhang Q, Hu LQ, Li HQ, et al. Beneficial effects of andrographolide in a rat model of autoimmune myocarditis and its effects on PI3K/Akt pathway. Korean J Physiol Pharmacol. 2019;23:103-111. doi:10.4196/kjpp.2019.23.2.103

44. Liang E, Liu X, Du Z, et al. Andrographolide ameliorates diabetic cardiomyopathy in mice by blockage of oxidative damage and NF- $\kappa$ B-mediated inflammation. Oxid Med Cell Longev. 2018;2018:1-13. doi:10.1155/2018/9086747

45. Lin KH, Marthandam Asokan S, Kuo WW, et al. Andrographolide mitigates cardiac apoptosis to provide cardio-protection in high-fatdiet-induced obese mice. Environ Toxicol. 2020;35:707-713. doi:10.1002/tox. 22906

46. Shu J, Huang R, Tian Y, et al. Andrographolide protects against endothelial dysfunction and inflammatory response in rats with coronary heart disease by regulating ppar and $\mathrm{nf}-\mathrm{\kappa b}$ signaling pathways. Ann Palliat Med. 2020;9:1965-1975. doi:10.21037/apm-20-960

47. Fujiwara N, Kobayashi K. Macrophages in inflammation. Curr Drug Targets Inflamm Allergy. 2005;4:281-286. doi:10.2174/156801005 4022024 
48. Li J, Piskol R, Ybarra R, et al. CD3 bispecific antibody-induced cytokine release is dispensable for cytotoxic $\mathrm{T}$ cell activity. Sci Transl Med. 2019;11:508.

49. Kitchens RL. Role of CD14 in cellular recognition of bacterial lipopolysaccharides. Chem Immunol. 2000;74:61-82.

50. Granucci F, Zanoni I. Role of CD14 in host protection against infections and in metabolism regulation. Front Cell Infect Microbiol. 2013;3:32.

51. Schwenger KJP, Chen L, Chelliah A, et al. Markers of activated inflammatory cells are associated with disease severity and intestinal microbiota in adults with non-alcoholic fatty liver disease. Int $\mathrm{J} \mathrm{Mol}$ Med. 2018;42:2229-2237.

52. Chistiakov DA, Killingsworth MC, Myasoedova VA, et al. CD68/ macrosialin: not just a histochemical marker. Lab Invest. 2017;97:4-13. doi:10.1038/labinvest.2016.116

53. Sansom DM, Manzotti CN, Zheng Y. What's the difference between CD80 and CD86? Trends Immunol. 2003;24:314-319. doi:10.1016/ S1471-4906(03)00111-X

54. Ade N, Antonios D, Kerdine-Romer S, et al. NF-kappaB plays a major role in the maturation of human dendritic cells induced by NiSO(4) but not by DNCB. Toxicol Sci. 2007;99:488-501. doi:10.1093/toxsci/kfm178

55. Suzuki Y, Shirai M, Asada K, et al. Macrophage mannose receptor, CD206, predict prognosis in patients with pulmonary tuberculosis. Sci Rep. 2018;8:13129. doi:10.1038/s41598-018-31565-5

56. Perkins ND. Integrating cell-signalling pathways with NF-kappaB and IKK function. Nat Rev Mol Cell Biol. 2007;8:49-62. doi: $10.1038 / \mathrm{nrm} 2083$

57. Hemmings BA, Restuccia DF. PI3K-PKB/Akt pathway. Cold Spring Harb Perspect Biol. 2012;4:a011189-a011189. doi:10.1101/cshperspect.a011189

58. Kaminska B. MAPK signalling pathways as molecular targets for anti-inflammatory therapy-from molecular mechanisms to therapeutic benefits. Biochim Biophys Acta. 2005;1754:253-262. doi:10.1016/ j.bbapap.2005.08.017

59. Feng B, Zhang Q, Wang X, et al. Effect of andrographolide on gene expression profile and intracellular calcium in primary rat myocardium microvascular endothelial cells. J Cardiovasc Pharmacol. 2017;70:369-381. doi:10.1097/FJC.0000000000000528

60. Pizzino G, Irrera N, Cucinotta M, et al. Oxidative stress: harms and benefits for human health. Oxid Med Cell Longev. 2017;2017:8416763. doi:10.1155/2017/8416763

61. Ansley DM, Wang B. Oxidative stress and myocardial injury in the diabetic heart. J Pathol. 2013;229:232-241. doi:10.1002/path.4113

62. Habtemariam S. The Nrf2/HO-1 axis as targets for flavanones: neuroprotection by pinocembrin, naringenin, and eriodictyol. Oxid Med Cell Longev. 2019;2019:4724920. doi:10.1155/2019/4724920

63. Teringova E, Tousek P. Apoptosis in ischemic heart disease. J Transl Med. 2017;15:87. doi:10.1186/s12967-017-1191-y

64. Mishra PK, Adameova A, Hill JA, et al. Guidelines for evaluating myocardial cell death. Am J Physiol Heart Circ Physiol. 2019;317: H891-h922. doi:10.1152/ajpheart.00259.2019

65. Elmore S. Apoptosis: a review of programmed cell death. Toxicol Pathol. 2007;35:495-516. doi:10.1080/01926230701320337

66. Charlier E, Relic B, Deroyer C, et al. Insights on molecular mechanisms of chondrocytes death in osteoarthritis. Int $J$ Mol Sci. 2016;17:2146. doi:10.3390/ijms17122146
67. Kantari C, Walczak H. Caspase- 8 and Bid: caught in the act between death receptors and mitochondria. Biochim Biophys Acta. 2011;1813:558-563. doi:10.1016/j.bbamcr.2011.01.026

68. Troncoso R, Ibarra C, Vicencio JM, et al. New insights into IGF-1 signaling in the heart. Trends Endocrinol Metab. 2014;25:128-137. doi:10.1016/j.tem.2013.12.002

69. Gao J, Liu Q, Xu Y, et al. PPAR $\alpha$ induces cell apoptosis by destructing Bcl2. Oncotarget. 2015;6:44635-44642. doi:10.18632/ oncotarget.5988

70. Ma ZG, Yuan YP, Wu HM, et al. Cardiac fibrosis: new insights into the pathogenesis. Int J Biol Sci. 2018;14:1645-1657. doi:10.7150/ ijbs. 28103

71. Rerolle JP, Hertig A, Nguyen G, et al. Plasminogen activator inhibitor type 1 is a potential target in renal fibrogenesis. Kidney Int. 2000;58:1841-1850. doi:10.1111/j.1523-1755.2000.00355.x

72. Thorin E, Webb DJ. Endothelium-derived endothelin-1. Pflugers Arch. 2010;459:951-958. doi:10.1007/s00424-009-0763-y

73. Trane AE, Pavlov D, Sharma A, et al. Deciphering the binding of caveolin-1 to client protein endothelial nitric-oxide synthase (eNOS): scaffolding subdomain identification, interaction modeling, and biological significance. $J$ Biol Chem. 2014;289:13273-13283. doi:10.1074/jbc.M113.528695

74. Sakila S, Begum N, Kawsar S, et al. Relationship of anti-fertility effects of Andrographis paniculata and hormonal assay in female rats. Bangladesh J Med Sci. 2009;3:10-14.

75. Worasuttayangkurn L, Nakareangrit W, Kwangjai J, et al. Acute oral toxicity evaluation of Andrographis paniculata-standardized first true leaf ethanolic extract. Toxicol Rep. 2019;6:426-430. doi:10.1016/j. toxrep.2019.05.003

76. Al Batran R, Al-Bayaty F, Al-Obaidi MMJ, et al. Acute toxicity and the effect of andrographolide on Porphyromonas gingivalis-induced hyperlipidemia in rats. Biomed Res Int. 2013;2013:594012. doi:10.1007/s12223-017-0496-9

77. Thamlikitkul V, Dechatiwongse T, Theerapong S, et al. Efficacy of Andrographis paniculata, Nees for pharyngotonsillitis in adults. J Med Assoc Thai. 1991;74:437-442.

78. Calabrese C, Berman $\mathrm{SH}$, Babish JG, et al. A phase I trial of andrographolide in HIV positive patients and normal volunteers. Phytother Res. 2000;14:333-338. doi:10.1002/1099-1573(200008) 14:5<333::AID-PTR584>3.0.CO;2-D

79. Panossian A, Hovhannisyan A, Mamikonyan G, et al. Pharmacokinetic and oral bioavailability of andrographolide from Andrographis paniculata fixed combination Kan Jang in rats and human. Phytomedicine. 2000;7:351-364. doi:10.1016/S09447113(00)80054-9

80. Bera R, Ahmed SK, Sarkar L, et al. Pharmacokinetic analysis and tissue distribution of andrographolide in rat by a validated LC-MS/ MS method. Pharm Biol. 2014;52:321-329. doi:10.3109/ 13880209.2013.836544

81. Sharma M, Sharma R. Identification, purification and quantification of andrographolide from Andrographis paniculata (Burm. F.) Nees by HPTLC at different stages of life cycle of crop. J Curr Chem Pharm Sci. 2013;3:23-32.

82. Pandey A, Gulati S, Gupta A, et al. Variation in andrographolide content among different accessions of Andrographis paniculata. Pharma Innovation J. 2019;8:140-144. 


\section{Publish your work in this journal}

Drug Design, Development and Therapy is an international, peerreviewed open-access journal that spans the spectrum of drug design and development through to clinical applications. Clinical outcomes, patient safety, and programs for the development and effective, safe, and sustained use of medicines are a feature of the journal, which has also been accepted for indexing on PubMed Central. The manuscript management system is completely online and includes a very quick and fair peer-review system, which is all easy to use. Visit http://www. dovepress.com/testimonials.php to read real quotes from published authors. 